\title{
El carácter polisémico de educación de calidad en el nivel universitario: una aproximación desde sus actores principales
}

\author{
The Polysemic Character of Quality Education at the University Level: An \\ Approach from Its Main Actors
}

\section{O caráter polissêmico da educação de qualidade no nível universitário: uma abordagem a partir de seus principais atores}

\author{
Miguel Ángel Clara Zafra \\ Universidad Veracruzana, Facultad de Contaduría y Administración, México \\ mclara@uv.mx \\ https://orcid.org/0000-0001-8152-0507 \\ César Vega Zárate \\ Universidad Veracruzana, Facultad de Contaduría y Administración, México \\ cevega@uv.mx \\ https://orcid.org/0000-0002-0233-4536
}

\section{Resumen}

Este estudio muestra los resultados derivados de una investigación exploratoria cuyo propósito fue analizar la percepción de diversos actores sobre la noción de calidad inserta en el ámbito de la educación, particularmente en un nivel universitario. Para ello, se realizó un estudio de carácter cuantitativo con alcance correlacional y de corte transversal. Luego de seguir un muestreo de tipo no probabilístico por conveniencia, se obtuvo un total de 763 sujetos categóricos. Los resultados indican que $59.8 \%$ de los encuestados considera que el principal atributo para referirse a una educación de calidad debe ser el proceso de enseñanza-aprendizaje, además de que la evaluación de la calidad en las instituciones de educación superior debe realizarse a través de los procesos de acreditación y certificación que realizan los organismos evaluadores. Posteriormente, se obtuvieron los análisis correlacionales a través de un análisis de correspondencias múltiples (ACM). Las percepciones de los diversos actores categóricos son divergentes y 


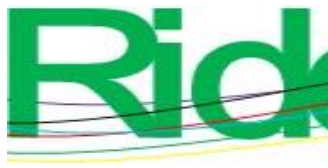

Revista Iberoamericana para la Investigación y el Desarrollo Educativo ISSN $2007-7467$

varían según el contexto donde se desarrollan, lo cual indica que aun estudiando a los principales actores universitarios, la educación de calidad es polisémica. Finalmente, se sugiere dirigir el debate hacia la construcción de una definición transdisciplinar que conjunte las diversas perspectivas.

Palabras clave: certificaciones y acreditaciones, educación de calidad, educación superior.

\section{Abstract}

This study shows the results derived from an exploratory research whose purpose was to analyze the perception of various actors on the notion of quality inserted in the field of education, particularly at a university level. To do this, a quantitative study with a correlational and cross-sectional scope was carried out. After following a nonprobabilistic sampling for convenience, a total of 763 categorical subjects were obtained. The results indicate that $59.8 \%$ of those surveyed consider that the main attribute to refer to a quality education should be the teaching-learning process, in addition to that the evaluation of quality in higher education institutions should be carried out through the accreditation and certification processes carried out by the evaluating bodies. Subsequently, the correlational analyzes were obtained through a multiple correspondence analysis (MCA). The perceptions of the various categorical actors are divergent and vary according to the context in which they develop, which indicates that even after studying the main university actors, quality education is polysemic. Finally, it is suggested to direct the debate towards the construction of a transdisciplinary definition that brings together the various perspectives.

Keywords: certifications and accreditations, quality education, higher education.

\section{Resumo}

Este estudo apresenta os resultados derivados de uma pesquisa exploratória cujo objetivo foi analisar a percepção de diversos atores sobre a noção de qualidade inserida no campo da educação, em particular no nível universitário. Para tanto, foi realizado um estudo quantitativo de abrangência correlacional e transversal. Depois de seguir uma amostragem não probabilística por conveniência, um total de 763 sujeitos categóricos foram obtidos. Os resultados indicam que $59.8 \%$ dos respondentes consideram que o principal atributo para se referir a uma educação de qualidade deve ser o processo de ensino-aprendizagem, além do fato de que a avaliação da qualidade nas instituições de 
ensino superior deve ser realizada por meio do credenciamento e processos de certificação levados a cabo pelos organismos avaliadores. Posteriormente, as análises correlacionais foram obtidas por meio de uma análise de correspondência múltipla (ACM). As percepções dos diversos atores categóricos são divergentes e variam de acordo com o contexto em que se desenvolvem, o que indica que, mesmo estudando os principais atores universitários, a educação de qualidade é polissêmica. Por fim, sugere-se direcionar o debate para a construção de uma definição transdisciplinar que reúna as várias perspectivas.

Palavras chave: certificações e acreditações, educação de qualidade, ensino superior. Fecha Recepción: Enero 2021

Fecha Aceptación: Julio 2021

\section{Introducción}

Actualmente, debido principalmente a la globalización, la noción de calidad ha adquirido un rol protagónico en el contexto de la educación superior como instrumento valioso para la mejora continua de los procesos educativos. Prueba de lo anterior, ha sido la creación de sistemas de evaluación, certificación y acreditación a nivel mundial. Sin embargo, por su carácter polisémico, también ha sido un concepto detonador de varios debates. En efecto, en la esfera educativa el empleo de este término ha sido fuertemente criticado y debatido por su naturaleza conceptual tan variable y maleable.

Para Lago, Gamoba y Montes (2014), calidad es un concepto complejo de definir, y quizá a raíz de lo anterior, es concebido de múltiples formas por los sujetos. Huelga decir que en la definición de cualquier término intervienen diversos factores como la cultura, el contexto y las propias preconcepciones de aquello que se está definiendo.

Schiefelbein y Schiefelbein (2000), Cubillo y Rozo (2009) y Mayo, Loredo y Reyes (2015) mencionan que la calidad suele ser asociada a términos como excelencia, mejora, eficiencia, eficacia, servicio al cliente, satisfacción, indicadores, acreditaciones y certificaciones, entre otros. Pareciera llevar consigo una especie de oxímoron: si bien es un concepto que puede ser visualizado fácilmente, es complejo de definir. Cabe destacar que proviene del contexto empresarial, donde fue inicial y extensamente tratado, y que con su llegada las instituciones educativas, sobre todo las universidades, han sumado a su agenda el cumplimiento de indicadores y procesos de evaluación.

Orozco, Olaya y Villate (2009) argumentan que la educación de calidad no debe circunscribirse únicamente a los logros cognitivos de los estudiantes o a la actualización de programas de estudios y procesos de aprendizaje, sino que debe ser un proceso más 


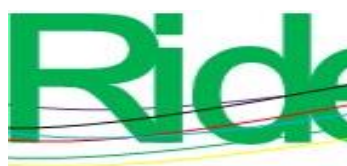

Revista Iberoamericana para la Investigación y el Desarrollo Educativo ISSN 2007 - 7467

diverso e integral en el que intervengan las necesidades de la sociedad y las demandas de los diversos dominios sociales: económico, político, cultural, religioso y administrativo.

Con base en lo antes descrito, la presente investigación se planteó como objetivo analizar la percepción sobre educación de calidad en el nivel superior a partir de sujetos categóricos: estudiantes de licenciatura y posgrado, profesores universitarios, empresarios y empleados en el contexto latinoamericano. La variable central es Educación de Calidad. Como ya dijimos, es un término polisémico y polivalente (Harvey y Green, 1993), además de poseer una combinación de funcionalidad, eficacia y eficiencia altamente correlacionada con la excelencia (Pérez, López, Peralta y Municio, 2000). La hipótesis que se planteó fue la siguiente: los diversos sujetos categóricos poseen una percepción divergente acerca de la educación de calidad en el nivel superior, lo cual denota la polisemia del término y sus diversas apreciaciones por los actores que intervienen en el sector universitario.

\section{Revisión de literatura}

La educación de calidad es aquella que realiza cambios significativos en el estudiante y enriquece su formación profesional (González y Espinoza, 2008). Para Bianchetti (2009), la calidad se encuentra vinculada a las características y funciones que debe cumplir la educación; es una herramienta de formación para el ejercicio ciudadano, y debe adaptarse a las demandas requeridas de la sociedad, del contexto económico y político, con el propósito de potenciar el desarrollo en cada país. Por su parte, Avendaño, Paz y Parada (2016) mencionan que calidad hace referencia a la mejora de los procesos educativos y pedagógicos donde el centro de la atención es el aprendizaje para el desarrollo humano.

De vuelta siguiendo a Bianchetti (2017), el debate entorno a la calidad en el contexto de la educación superior surge de la relatividad de su definición, lo cual implica una controversia en lo académico y público, y hace alusión a que los diferentes actores que intervienen poseen diversas percepciones acerca de este tema. Lo anterior refuerza el posicionamiento teórico de Harvey y Green (1993) acerca de que educación de calidad es un término polisémico, confuso y escurridizo. En la tabla 1 se mencionan las características principales según algunos de los autores antes mencionados. 
Tabla 1. Concepciones y características de la calidad en el contexto de la educación superior

\begin{tabular}{|c|c|}
\hline $\begin{array}{l}\text { Concepción de } \\
\text { calidad }\end{array}$ & Características \\
\hline onal & $\begin{array}{l}\text { - Visión tradicional: basada en la distinción y la exclusividad; las } \\
\text { universidades las personalizan. } \\
\text { - Visión de excelencia: superación de los estándares. Se requieren } \\
\text { los mejores profesores, estudiantes, instalaciones, planes y } \\
\text { programas de estudios, y lograr todas las acreditaciones. } \\
\text { - Visión de cumplimiento de los estándares mínimos: cumplir todos } \\
\text { los indicadores, el estándar es el objetivo de la universidad. }\end{array}$ \\
\hline $\begin{array}{ll}\text { Perfección } & y \\
\text { consistencia } & \end{array}$ & $\begin{array}{l}\text { Se asocia con el pensamiento de cero defectos, hacer las cosas bien } \\
\text { a la primera vez. Posee una relación con la adopción de una cultura } \\
\text { de calidad. La universidad se encuentra constituida por } \\
\text { departamentos, cada uno de estos debe de cumplir con su trabajo en } \\
\text { el proceso que le fue asignado. }\end{array}$ \\
\hline $\begin{array}{l}\text { Adecuación a } \\
\text { una finalidad }\end{array}$ & $\begin{array}{l}\text { La calidad se asocia como el ajuste a un propósito. En este apartado } \\
\text { la calidad se evalúa a través del resultado final. Se vincula con la } \\
\text { satisfacción de las necesidades de los clientes, por lo tanto, se } \\
\text { relaciona con la funcionalidad. } \\
\text { Se evalúa que la universidad cumpla con los objetivos que ha } \\
\text { establecido desde su propia misión y funciones. }\end{array}$ \\
\hline Valor por dinero & $\begin{array}{l}\text { La calidad se relaciona con la eficiencia económica, con los } \\
\text { objetivos obtenidos de una inversión, un enfoque que proviene de } \\
\text { la idea de la rendición de cuentas. }\end{array}$ \\
\hline Transformación & $\begin{array}{l}\text { Se plantea desde la función principal de la universidad, en este caso } \\
\text { en el desarrollo del estudiante y en su capacitación para la vida. En } \\
\text { este sentido, se orienta, más que en la vida profesional, hacia } \\
\text { acciones sobre la ética y compromiso social. }\end{array}$ \\
\hline
\end{tabular}

Fuente: Elaboración propia con base en Andreasen, Colombo, Mollo, Gilli y López 


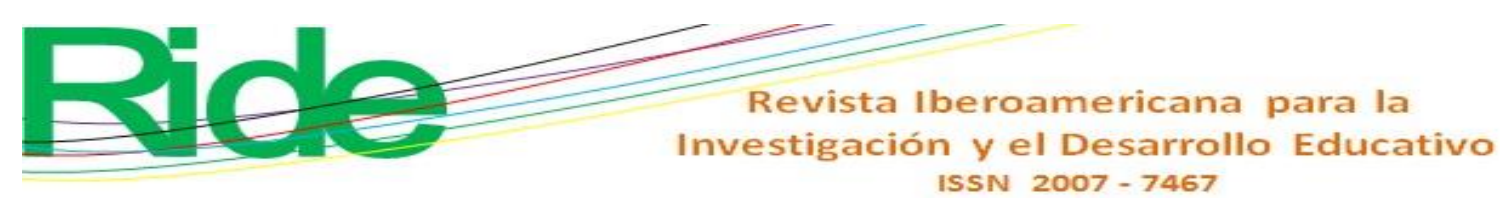

Así pues, la calidad en el ámbito universitario ha sido asociada o llegado a interpretarse como la excelencia, perfección, finalidad, valor económico y transformación. Frente a este amplio y complejo panorama, es necesario conjuntar las diversas perspectivas y dar con una definición transdisciplinaria.

Los sistemas educativos han colocado a la calidad como un tema primordial de la agenda pública, además de ser un elemento clave en la mejora del servicio educativo. De acuerdo con González y Espinoza (2008), las universidades latinoamericanas asocian la educación de calidad con los procesos de evaluación, certificación y acreditación que obedecen al aseguramiento de recursos públicos a través de la rendición de cuentas. Por el contrario, Fernández, Egido y Carballo (2016) argumentan que la calidad debe estar vinculada al proceso de enseñanza-aprendizaje, lo cual genera un impacto en la formación integral del estudiante.

Ahora bien, a raíz de los variados acercamientos a la calidad en la educación superior, han surgido diversas cadenas conceptuales, tales como educación de calidad, calidad en la educación y calidad educativa. ¿Son lo mismo o poseen el mismo sentido o noción? Orozco et al. (2009) indican que no, ya que cada cadena hace referencia a significados diferentes, aunque en la literatura son utilizados indistintamente e inclusive empleados como sinónimos. Una vez más se pone de manifiesto lo polisémico de la noción de calidad en el contexto de la educación superior (Harvey y Green, 1993).

Sin duda, en el contexto académico, la calidad es compleja de definir e interpretar, lo cual, como se ha mencionado, se debe a la propia polivalencia del término y a las diversas percepciones de los diversos actores. Garzón (2013) indica que es necesario observar estas, las percepciones de los diversos actores interesados en el tema de la calidad, con el propósito de conocer sus posturas (tabla 2). 
Tabla 2. Percepciones acerca de la educación de calidad de diversos actores

\begin{tabular}{|c|c|}
\hline Actores & Percepción \\
\hline Estudiantes & $\begin{array}{l}\text { La calidad es un sinónimo de excelencia y se verá } \\
\text { interpretado en el logro de las competencias adquiridas } \\
\text { o desarrolladas en la universidad que coadyuvarán en el } \\
\text { desarrollo de su desempeño profesional y ciudadano. }\end{array}$ \\
\hline Instituciones privadas & $\begin{array}{l}\text { Se evaluará la calidad como un retorno de inversión y } \\
\text { marca institucional, el posicionamiento de la misma } \\
\text { universidad, y su orientación hacia la mercantilización. }\end{array}$ \\
\hline Instituciones públicas & $\begin{array}{l}\text { Se interpreta la calidad desde el logro de alcanzar una } \\
\text { mayor cobertura de la educación superior a un menor } \\
\text { costo, la idea es completamente inversa a las privadas. }\end{array}$ \\
\hline Sistema productivo & $\begin{array}{l}\text { La calidad se asocia con las competencias coherentes, } \\
\text { específicas y profesionales del capital humano para con } \\
\text { las demandas del sistema. }\end{array}$ \\
\hline Directores & $\begin{array}{l}\text { Es sinónimo de coherencia del sistema educativo } \\
\text { normativo con los objetivos de las propias } \\
\text { universidades. Este conjunto de sujetos asocia la calidad } \\
\text { al cumplimiento de indicadores y el logro de } \\
\text { acreditaciones por parte de su institución. }\end{array}$ \\
\hline Profesores e investigadores & $\begin{array}{l}\text { La calidad se asocia a la mejora del proceso de } \\
\text { enseñanza-aprendizaje. Además, debaten sobre la } \\
\text { coherencia del sistema educativo con la práctica } \\
\text { docente. }\end{array}$ \\
\hline $\begin{array}{lll}\text { Actores del proceso } \\
\text { educativo }\end{array}$ & $\begin{array}{l}\text { Se valora como el grado de satisfacción de sus } \\
\text { necesidades y expectativas personales, lo asocian a la } \\
\text { calidad en el servicio que la universidad brinda a la } \\
\text { sociedad. }\end{array}$ \\
\hline Sistema educativo & $\begin{array}{l}\text { El sistema educativo asocia, evalúa y aplica la calidad } \\
\text { desde las propias políticas orientadas a la mejora de la } \\
\text { educación. Por lo tanto, se promueve y crea un sistema } \\
\text { de evaluación y acreditación de las instituciones y sus } \\
\text { programas académicos y demanda del sector productivo } \\
\text { a las universidades, la gestión de la calidad al interior de }\end{array}$ \\
\hline
\end{tabular}


sus instituciones, y todo lo relacionado con la mejora de los procesos.

Fuente: Elaboración propia con base en Garzón (2013)

En el nivel superior, la calidad ha tomado un rol sobresaliente y se ha incorporado intrínsecamente a las funciones sustantivas de las universidades. Esto como respuesta a los requerimientos de la política educativa de cada país. Consecuentemente, se han creado los sistemas de evaluación, certificación y acreditación para asegurar la calidad en las universidades.

Complementando lo anterior, Lugo e Ithurburu (2019) sostienen que no solo las instituciones educativas son las responsables en mejorar la educación, sino también es responsabilidad del Estado, puesto que su labor es diseñar, implementar y evaluar políticas públicas en materia de educación de acuerdo con las necesidades de cada región. Para López (2007), el diseño de políticas públicas en este rubro debe estar enfocado a mejorar la calidad y la pertinencia de las instituciones educativas con la finalidad de potencializar el desarrollo de cada país.

Lorente (2016) indica que sigue siendo un reto ofrecer una educación de calidad en toda la región de América Latina. Aunque se han priorizado los resultados, los estándares y criterios de los modelos de calidad no se encuentran bien definidos en la actualidad. Por tanto, se requiere que el debate sobre la educación en esta región se dirija hacia un consenso que lleve a potenciar el desarrollo educativo. Por su parte, Clara y Vega (2020) afirman que la educación de calidad es clave para el desarrollo, pero su contribución dependerá de las mejoras que se realicen a los sistemas educativos y de la comprensión de las implicaciones de la propia calidad en las universidades.

Cabe destacar que existen diversas investigaciones nacionales e internacionales que buscan conocer las diversas percepciones en relación con el tema de la educación de calidad. Duque y Chaparro (2012), a través de una encuesta a estudiantes en Colombia, indagaron la percepción sobre la calidad en el servicio. Según los resultados obtenidos, los estudiantes asocian la calidad funcional al servicio que les presta la institución.

Por otro lado, Bernasconi y Rodríguez (2018) llevaron a cabo un estudio en Chile a directivos de universidades, quienes plantearon la calidad desde la perspectiva de la evaluación. Mientras que la investigación de Araya (2013) sobre la calidad en la educación superior arrojó que esta se asocia y se percibe desde diversos horizontes: el mercado, los estudiantes y la sociedad.

En México, los estudios de Alvarado, Luyando y Picazzo (2015) y Alvarado, Morales y Aguayo (2016) encontraron algunos hallazgos fundamentales al respecto: los 


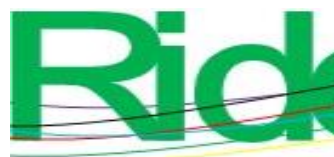

Revista Iberoamericana para la Investigación y el Desarrollo Educativo ISSN $2007-7467$

estudiantes, principales actores en este proceso, asocian la calidad hacia la mejora del servicio educativo. Por último, Salas (2013) realizó un estudio donde reafirma que la calidad se puede interpretar desde muchas percepciones; en el caso de la acreditación, esta posee un impacto en la gestión institucional de las universidades y en sus actores. Además, subraya que la calidad en las universidades genera un efecto en todas las organizaciones fuera del contexto universitario, prueba de ello son las demandas de personal calificado y especializado que requieren los sectores económico, empresarial y público.

\section{Metodología}

El procedimiento metodológico tuvo un enfoque cuantitativo, con un alcance descriptivo-correlacional y de corte transversal (Hernández, Fernández y Baptista, 2014). La recolección de datos fue por medio de una encuesta de 14 preguntas cerradas y lanzada a través de la plataforma de Google Drive durante el periodo febrero-mayo de 2020. Los informantes fueron los sujetos categóricos descritos anteriormente. La técnica estadística empleada fue un análisis de correspondencia múltiples (ACM) con el software estadístico SPSS 24.

\section{Muestra}

Para el presente estudio, se empleó un muestreo no probabilístico que combinó el de conveniencia e intencional (Otzen y Manterola, 2017). La distribución del cuestionario se realizó a través de Google Drive. Se obtuvo un total de 763 participantes. Geográficamente, 90.3 \% corresponde a sujetos de México y el resto a otros países de Latinoamérica. En cuestión de género, $54.2 \%$ son hombres y $45.8 \%$ mujeres. Respecto a la edad, la mayoría (60.4 \%) tiene una edad que oscila entre los 17 y 30 años. En el caso de la ocupación, existe una distribución equitativa.

\section{Instrumento de recopilación}

El cuestionario empleado fue denominado Percepción sobre educación de calidad en la educación superior. Se diseñó de acuerdo con lo establecido por Supo (2012). Se aplicó una prueba piloto y derivado de ello se modificó el cuestionario. En total, se compuso de 14 preguntas, de las cuales cuatro fueron preguntas sobre las caracteríticas generales de los individuos (sexo, edad, ocupación y nacionalidad) y 10, de tipo nominal, sobre la percepción acerca de la educación de calidad en el contexto de la educación superior. 


\section{Resultados}

En el presente apartado se presentan los hallazgos más importantes respecto al propósito del estudio en cuestión. En primer lugar, se informa acerca de los resultados descriptivos que corresponden al análisis exploratorio y, en segundo, sobre el análisis correlacional que corresponde al ACM.

\section{Análisis exploratorio}

Respecto a qué entienden por calidad, $42.3 \%$ de los participantes asocia el término a la excelencia, $17.4 \%$ a la satisfacción y $17.6 \%$ al cumplimiento de requisitos. Por otro lado, referente a la educación, 45 \% lo relaciona como un proceso de formación y $11 \%$ a herramientas para la vida. Lo anterior fue la pauta para cuestionar sobre qué entienden por educación de calidad. Aquí $39.2 \%$ asocia este concepto al logro eficiente de competencias, $28.4 \%$ a la excelencia educativa y $18.7 \%$ a la mejora del proceso educativo.

Aunado a ello, se preguntó sobre los atributos que deben considerarse para una educación de calidad: $59.8 \%$ considera que lo central debe ser el proceso de enseñanzaaprendizaje y $20.1 \%$ los planes o programas de estudio. Sobre los aspectos que deben considerar las instituciones de educación superior para ofrecer una educación de calidad, $43.8 \%$ reafirma la importancia del proceso de enseñanza-aprendizaje y $31.2 \%$ el desarrollo integral del estudiante. Además, considerando el protagonismo que han tomado las acreditaciones en los procesos de evaluación de la calidad en las instituciones de educación superior, se cuestionó si estaban de acuerdo con que se evalúe la calidad a través de estas, y $43.1 \%$ y $35.5 \%$ estuvieron parcial y totalmente de acuerdo, respectivamente.

Por otro lado, considerando la relevancia de la calidad en el nivel superior, se preguntó dónde tiene mayor impacto una educación de calidad: $45.2 \%$ opinó que en el desarrollo y formación del profesionistas, $19.7 \%$ que en el desarrollo de cada país y $17.4 \%$ que en el mercado laboral. Respecto a qué aspectos deben considerarse para mejorar la calidad de la educación, $31.6 \%$ enfatizó en el proceso de enseñanzaaprendizaje, $26.3 \%$ en los planes y programas de estudio y $16 \%$ en las políticas públicas. Y respecto a si las acreditaciones de las instituciones de educación superior contribuyen a generar una educación de calidad, 80.3 \% opinó que sí contribuyen.

Por último, se cuestionó sobre si los términos educación de calidad, calidad de la educación y calidad educativa son sinónimos y 39.8 \% respondió que no, 33.4 \% que sí y $26.7 \%$ que tal vez. 


\section{Análisis correlacional}

Para el análisis correlacional, se plantea, en primer lugar, considerar la pregunta 10 como variable clave que mide la polisemia de educación de calidad. Teniendo esto en consideración, se procedió a realizar la prueba de hipótesis a través del cálculo de ji al cuadrado, que se utiliza para variables de tipo nominal. El procedimiento consistió en considerar la variable Polisemia con el resto de las variables que integran el estudio. Derivado de ello, se encontró que la variable Polisemia guarda una relación significativa con Edad, Ocupación, Educación, Acreditación y Contribución de las Certificaciones y Acreditaciones.

En la tabla 3 se aprecia que los sujetos de 20 a 30 años son los que en mayor proporción (31.8 \% y $37.3 \%$, respectivamente) asumen que la educación de calidad es polisémica; caso contrario sucede con los que tienen entre 31-60 años. Además, se aprecia un resultado de $\chi^{2}=67.527$ y P-valor de 0.000 , lo cual indica que se rechaza la hipótesis nula y que existe dependencia entre estas variables.

Tabla 3. Prueba ji al cuadrado entre Polisemia y Edad

\begin{tabular}{|c|c|c|c|c|c|}
\hline \multirow{2}{*}{\multicolumn{2}{|c|}{ Variable* }} & \multicolumn{4}{|c|}{ Polisemia de educación de calidad } \\
\hline & & \multirow{2}{*}{$\begin{array}{l}\text { Sí } \\
31.8\end{array}$} & \multirow{2}{*}{$\begin{array}{l}\text { No } \\
14.8\end{array}$} & \multirow{2}{*}{$\begin{array}{l}\text { Tal vez } \\
39.3\end{array}$} & \multirow{2}{*}{$\begin{array}{l}\text { Total } \\
27.0\end{array}$} \\
\hline \multirow{6}{*}{ Edad } & 20 años o menos & & & & \\
\hline & 21-30 años & 37.3 & 30.9 & 32.8 & 33.6 \\
\hline & 31-40 años & 11.8 & 19.4 & 14.2 & 15.5 \\
\hline & 41-50 años & 9.4 & 21.7 & 9.3 & 14.3 \\
\hline & 51-60 años & 5.9 & 9.2 & 3.4 & 6.6 \\
\hline & 61 años o más & 3.9 & 3.9 & 1.0 & 3.1 \\
\hline \multicolumn{2}{|l|}{ Total } & 100 & 100 & 100 & 100 \\
\hline \multicolumn{6}{|c|}{$\begin{array}{l}\text { Nota: los resultados se presentan en porcentajes. La prueba de hipótesis } \\
\text { se calculó a través de la ji al cuadrado: } \chi^{2}=67.527, \mathrm{df}=10, \mathrm{P} \text {-valor }= \\
0.000 \text {. }\end{array}$} \\
\hline
\end{tabular}

\section{Fuente: Elaboración propia}

En la tabla 4 se aprecia el análisis de contingencia entre la variable Polisemia y Ocupación. Los estudiantes de licenciatura, especialidad, bachillerato, empresarios y trabajadores son quienes apoyan que la educación es polisémica; los profesores universitarios y los estudiantes de posgrado son quienes opinan lo contrario. En la prueba de ji al cuadrado se obtuvo una $\chi^{2}=74.627$ y un P-valor de 0.000 , lo cual denota que se rechaza la hipótesis nula y que sí existe dependencia entre estas. 
Tabla 4. Prueba ji al cuadrado entre Polisemia y Ocupación

\begin{tabular}{|c|c|c|c|c|c|}
\hline & & Polis & de $e d$ & on de call & \\
\hline Variable* & & Sí & No & Tal vez & Total \\
\hline & E Bachillerato & 2.0 & 0.30 & 2.0 & 2.0 \\
\hline & E Licenciatura & 43.5 & 27.3 & 52.9 & 39.6 \\
\hline & E Especialidad & 4.7 & 2.6 & 3.9 & 3.7 \\
\hline & E Maestría & 1.6 & 6.3 & 2.9 & 3.8 \\
\hline Ocunación & E Doctorado & 1.2 & 3.0 & 2.0 & 2.1 \\
\hline & P Investigador & 3.5 & 7.6 & 2.0 & 4.7 \\
\hline & P Completo & 7.5 & 15.1 & 7.4 & 10.5 \\
\hline & P Asignatura & 8.2 & 13.8 & 4.9 & 9.6 \\
\hline & Empresario & 3.1 & 3.3 & 1.0 & 2.6 \\
\hline & Empleador & 0.8 & 1.6 & 0.5 & 1.0 \\
\hline & Trabajador & 23.9 & 19.1 & 20.6 & 21.1 \\
\hline Total & & 100 & 100 & 100 & 100 \\
\hline $\begin{array}{l}\text { Nota: los re } \\
\text { prueba de h } \\
\text { valor }=0.00\end{array}$ & $\begin{array}{l}\text { s se presentan } \\
\text { se calculó a tr }\end{array}$ & rcen & $E=$ & $\begin{array}{l}\text { ntes, } P= \\
2=74.62\end{array}$ & $\begin{array}{l}\text { fesor. } \\
=20,\end{array}$ \\
\hline
\end{tabular}

\section{Fuente: Elaboración propia}

En la tabla 5, por su parte, se aprecian los valores cruzados entre la variable Polisemia y lo que los encuestados entienden por educación. En general, los actores que entienden la educación como la adquisición de conocimiento y como un proceso de formación apoyan la idea de la polisemia. Los valores de la asociación indican que $\chi^{2}=$ 23.837 y que el P-valor es 0.02, lo cual denota que existe relación entre estas variables. 
Tabla 5. Prueba ji al cuadrado entre Polisemia y Educación de Calidad

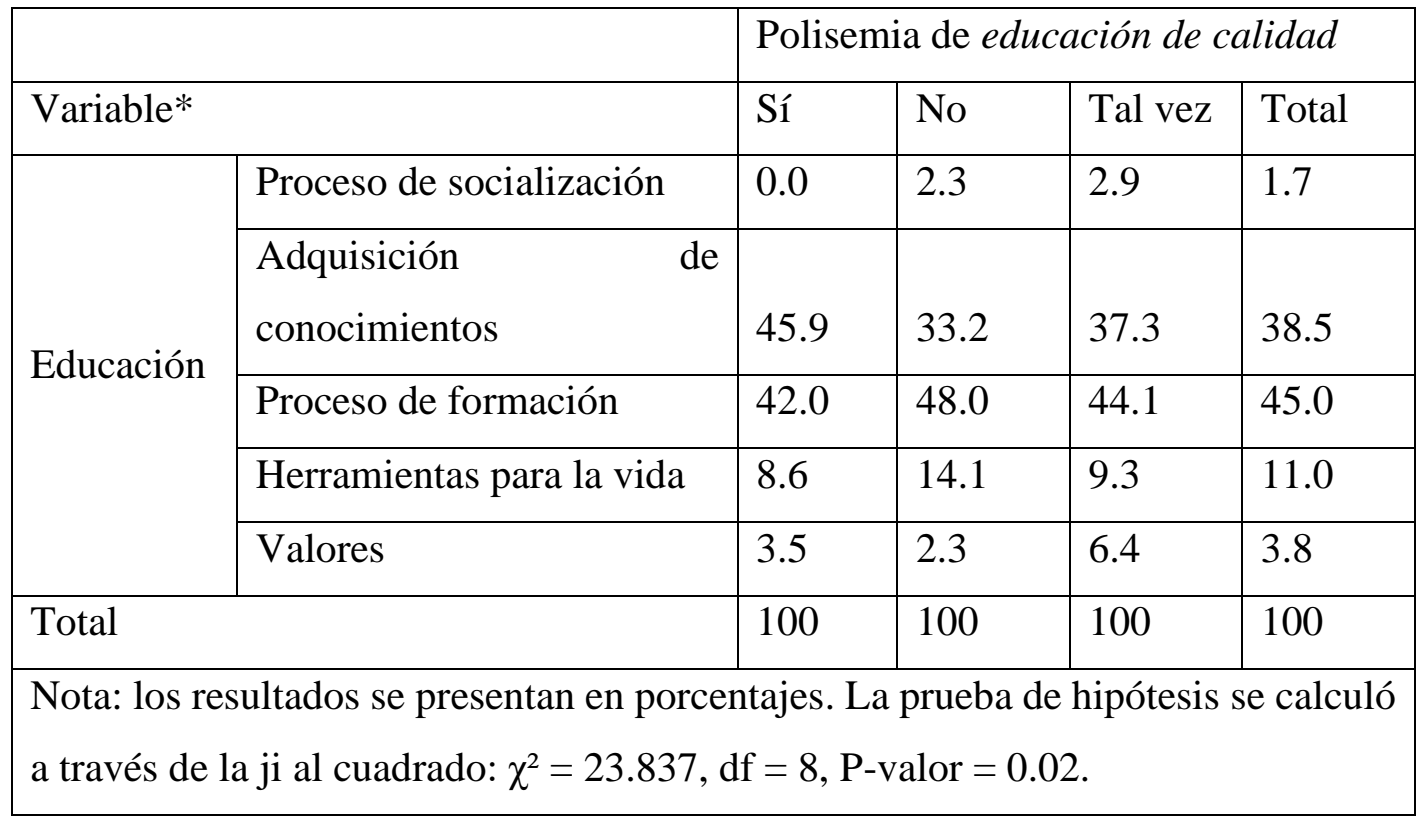

Fuente: Elaboración propia

En la tabla 6 se observan los valores de la contingencia entre las variables Polisemia y Acreditaciones y Certificaciones. Se aprecia que la mayoría (45.1\%) está de acuerdo en que las acreditaciones y certificaciones evalúen la educación de calidad; sin embargo, los datos demuestran estar de acuerdo con la polisemia. La prueba de ji al cuadrado dio como resultado $\chi^{2}=38.992$ y un $\mathrm{P}$-valor $=0.000$, lo cual denota la dependencia.

Tabla 6. Prueba ji al cuadrado entre Polisemia y Acreditaciones

\begin{tabular}{|c|c|c|c|c|c|}
\hline \multicolumn{2}{|l|}{ Variables } & \multicolumn{4}{|c|}{$\begin{array}{l}\text { Polisemia de educación de } \\
\text { calidad }\end{array}$} \\
\hline & & Sí & No & Tal vez & Total \\
\hline \multirow{5}{*}{$\begin{array}{l}\text { Acreditaciones } \\
\text { y } \\
\text { Certificaciones }\end{array}$} & Totalmente de acuerdo & 45.1 & 29.9 & 30.9 & 35.3 \\
\hline & Parcialmente de acuerdo & 40.0 & 40.5 & 51.1 & 43.1 \\
\hline & $\begin{array}{l}\mathrm{Ni} \text { de acuerdo ni en } \\
\text { desacuerdo }\end{array}$ & 11.4 & 15.8 & 13.2 & 13.6 \\
\hline & Parcialmente en desacuerdo & 2.4 & 8.9 & 3.9 & 5.4 \\
\hline & Totalmente en desacuerdo & 1.2 & 4.9 & 1.1 & 2.6 \\
\hline \multicolumn{2}{|l|}{ Total } & 100 & 100 & 100 & 100 \\
\hline \multicolumn{6}{|c|}{$\begin{array}{l}\text { Nota: los resultados se presentan en porcentajes. La prueba de hipótesis se } \\
\text { calculó a través de la ji al cuadrado: } \chi^{2}=38.992, \mathrm{df}=8 \mathrm{P} \text {-valor }=0.000 \text {. }\end{array}$} \\
\hline
\end{tabular}

Fuente: Elaboración propia 


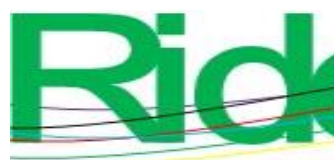

Revista Iberoamericana para la Investigación y el Desarrollo Educativo ISSN $2007-7467$

En la tabla 7 se aprecian los valores de la prueba de contingencia entre las variables relacionadas con la polisemia y si las certificaciones y acreditaciones contribuyen a generar una educación de calidad. Los datos indican que las acreditaciones y certificaciones sí contribuyen en la generación de una educación de calidad. Respecto a la prueba de ji al cuadrado, se aprecia una $\chi^{2}=28.419$ y un P-valor $=0.000$, lo que indica relación.

Tabla 7. Prueba ji al cuadrado entre Polisemia y Acreditaciones

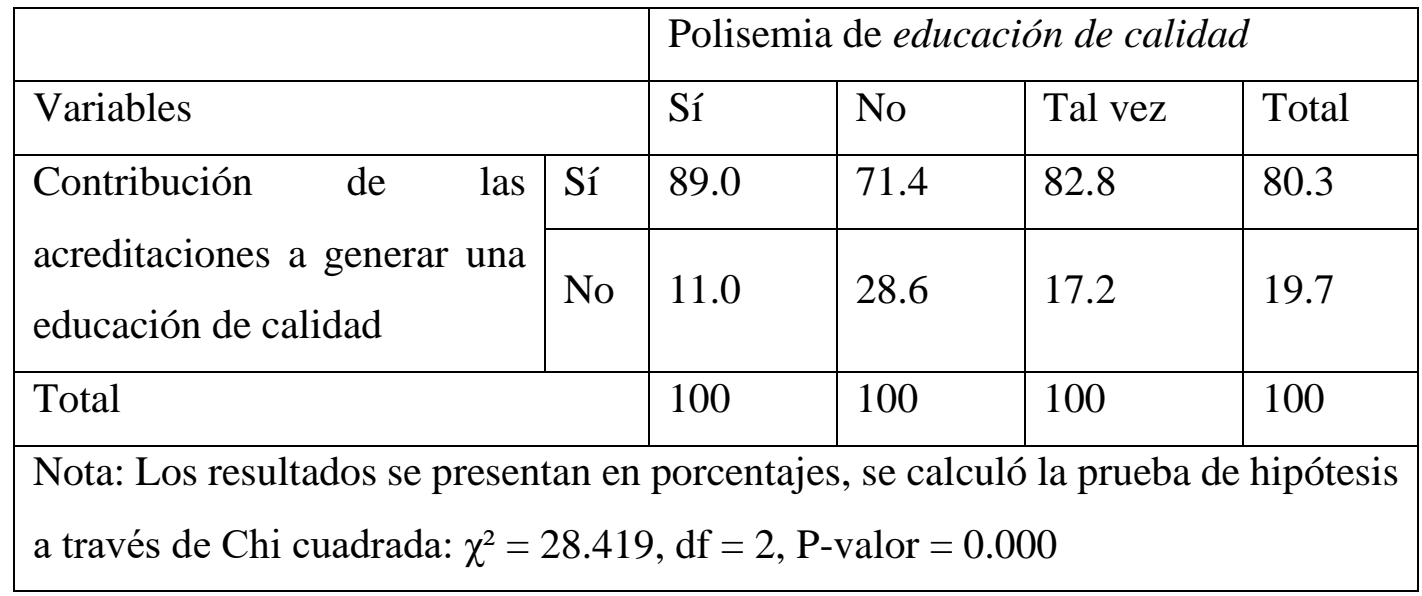

\section{Fuente: Elaboración propia}

Los resultados demuestran la dependencia entre las variables. Por tanto, se procedió a realizar el ACM (Greenacre, 2008). Y para ello, primeramente se efectuó el procesamiento del modelo de análisis (tabla 8).

Tabla 8. Resumen del modelo, varianza contabilizada

\begin{tabular}{|l|l|l|l|l|}
\hline Dimensión & $\begin{array}{l}\text { Alfa de } \\
\text { Cronbach }\end{array}$ & $\begin{array}{l}\text { Total } \\
\text { (autovalor) }\end{array}$ & $\begin{array}{l}\text { Inercia } \\
\text { varianza }\end{array}$ \\
\hline 1 & 0.660 & 2.221 & 0.370 & 37.015 \\
\hline 2 & 0.431 & 1.560 & 0.260 & 26.007 \\
\hline Total & & 3.781 & 0.630 & \\
\hline Media & $0.565^{\text {a }}$ & 1.891 & 0.315 & 31.511 \\
\hline Nota: la media de alfa de Cronbach se basa en la media de autovalor. \\
\hline
\end{tabular}

\section{Fuente: Elaboración propia}

Los datos del modelo de ACM permiten visualizar la varianza contabilizada, la cual se distribuye en dos dimensiones. La varianza de la dimensión uno es de 31.015 y de la dimensión dos es de 26.007, lo que implica que el modelo representa un 63.022 de la varianza del total de los datos obtenidos en el estudio. Para la construcción del gráfico de 
ACM se consideró la variable de Polisemia y todas las otras variables con las que guarda dependencia (antes mencionadas). En la figura 1 se aprecian las asociaciones.

Figura 1. Gráfico del ACM sobre la educación de calidad

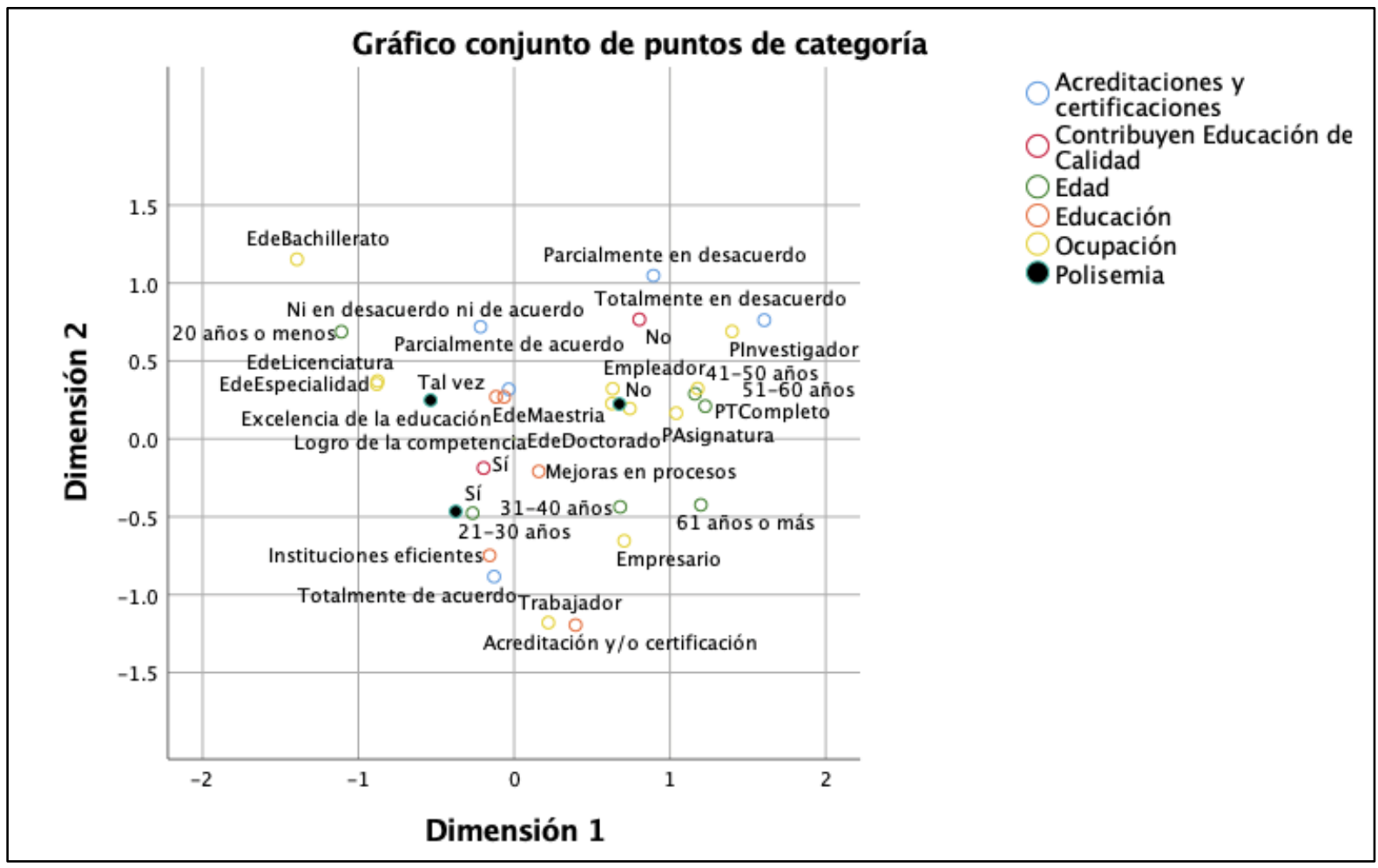

Fuente: Elaboración propia

La combinación de los ejes de la dimensión uno (horizontal) y dos (vertical) indican las asociaciones, y se muestra un poder explicativo muy alto, ya que se rebasa $63 \%$ de la variabilidad total de las variables. Los sujetos perciben que las diversas combinaciones de los términos educación y calidad son sinónimos.

Para los que respondieron "No", las concentraciones denotan asociación próxima con empleadores y estudiantes de posgrado, los que además se encuentran parcialmente de acuerdo con que las acreditaciones y certificaciones sean la forma para garantizar una educación de calidad. En este mismo sentido, se asocia también a profesores universitarios que se encuentran en un intervalo de edad de 41 años o más.

Para los sujetos que respondieron "Tal vez" se observa que se asocia a estudiantes de licenciatura y de especialidad, además de que estos asumen la educación como un proceso de adquisición de conocimientos y valores, y se encuentran parcialmente de acuerdo con que sean las acreditaciones y certificaciones el medio para evaluar la educación. Para los sujetos que respondieron "Sí”, el ACM indica que son trabajadores y empresarios que visualizan la educación como un proceso de formación y se encuentran totalmente de acuerdo con las acreditaciones en la generación de calidad en las 
instituciones de educación superior. En suma, las percepciones de la educación de calidad por los actores estudiados son variadas y es posible afirmar que esta percepción depende en gran medida de su ocupación y que a mayor grado de escolaridad poseerán mejor conocimiento sobre el tema.

Finalmente, cabe resaltar que las percepciones que se han podido describir denotan las divergencias respecto a una educación de calidad, sus implicaciones y su aplicación en las instituciones educativas.

\section{Discusión}

Los resultados demuestran la polisemia de la educación de calidad desde la percepción de diversos actores categóricos, lo cual denota a su vez la complejidad para definir dicho término y respalda el planteamiento teórico de la existencia de múltiples definiciones desde diversas perspectivas (Acosta, 2015; Harvey y Green, 1993). Tal y como Murillo y Román (2010) hacían alusión a que otros actores no universitarios deberían involucrarse, los resultados indican que es importante conocer su percepción, aunque se pone de relieve que estos desconocen las implicaciones de una educación de calidad.

Las diversas investigaciones antes citadas señalan el rol protagónico del estudiante en la educación de calidad, ya que finalmente es quien recibe el servicio educativo (Alvarado, Luyando et al., 2015; Alvarado, Morales et al., 2016). El análisis de los resultados indica que los estudiantes fueron quienes participaron en mayor medida, sin embargo, se pudo constatar que los de licenciatura, aunque se encuentran en el contexto universitario, desconocen las implicaciones de una educación de calidad, en cambio, los estudiantes de posgrado poseen mayor conocimiento sobre estos aspectos.

Los profesores universitarios poseen percepciones que convergen, sin embargo, en el tema de la evaluación son los profesores de asignatura, seguido de los de tiempo completo y por último los investigadores, quienes están de acuerdo con que las acreditaciones y certificaciones sean el medio para medir la calidad en el nivel universitario.

Contrario a lo mencionado por López (2007), quien considera de suma importancia que el diseño de políticas públicas se enfoque a mejorar la calidad de las instituciones educativas, los resultados de este estudio detallaron que la atención de las universidades debe centrarse en el proceso de enseñanza-aprendizaje, seguido por lo planes y programas de estudio y, por último, en el diseño de políticas públicas. Como se 


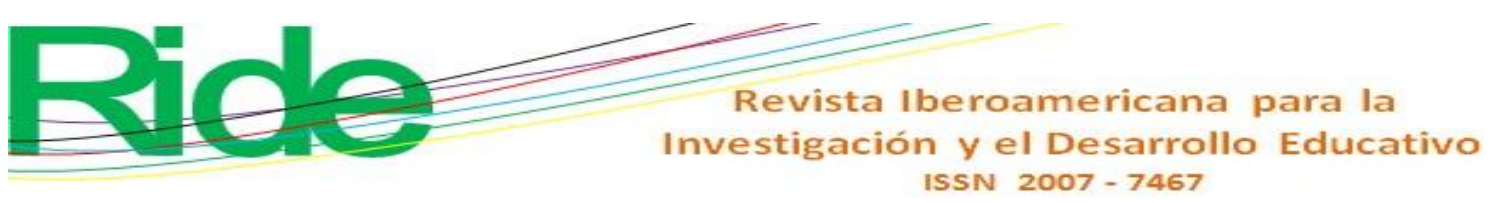

puede observar, los resultados de esta investigación tienen alguna similitud con algunos estudios, pero también presentan un contraste con otros.

\section{Fortalezas}

Las fortalezas del estudio radican en la presentación de los resultados a través de de un ACM, el cual visualmente permite identificar las divergentes percepciones por parte de los sujetos categóricos en relación con las implicaciones de una educación de calidad. Además, se presentan los resultados de los principales actores que intervienen de acuerdo con la revisión de la literatura.

\section{Debilidades}

Las principales debilidades del estudio radican en el análisis de las variables, debido a que son de tipo nominal y no permiten realizar un análisis más complejo.

\section{Conclusiones}

En esta investigación se aplicó una encuesta a diversos actores del contexto universitario; se buscó principalmente analizar la percepción sobre la educación de calidad en el nivel superior a partir de sujetos categóricos: estudiantes de licenciatura y posgrado, profesores universitarios, empresarios y empleados. Principalmente, el análisis consistió en un procesamiento descriptivo a través del cual se constató que existen diferencias en las percepciones de los sujetos categóricos. Posterior a ello, se procedió a realizar un ACM. Consecuentemente, se elaboraron las tablas de contingencia y el cálculo de ji al cuadrado para encontrar dependencias entre las variables.

El análisis descriptivo en general permitió conocer que los sujetos categóricos conciben a la educación de calidad como el logro eficiente de la competencia lograda por el estudiante, además de que el proceso de enseñanza-aprendizaje es el elemento central que deben considerar las universidades para garantizar este atributo en la educación. Igualmente, se lograron conocer otras percepciones que apuntan a que el desarrollo de profesionistas es el elemento donde más impacta una educación de calidad.

Por otro lado, a través del ACM se contrastaron las percepciones de los sujetos categóricos y se pudieron visualizar las divergencias. Los estudiantes de licenciatura y bachillerato se inclinan hacia la polisemia; por lo que respecta a los profesores y estudiantes de posgrados, estos no apoyan la polisemia, y por último, los actores no universitarios desconocen las implicaciones de la educación de calidad. En conclusión, 
las percepciones divergentes de los sujetos ponen de manifiesto la polisemia de educación de calidad e invita a reflexionar sobre las implicaciones de la calidad en las universidades.

\section{Futuras líneas de investigación}

Algunas de las líneas de investigación que surgen derivado de este estudio son: 1) analizar la educación de calidad con otro tipo de variables que permitan identificar la influencia entre estas, 2) elaborar una definición transdisciplinar sobre educación de calidad que busque conjuntar las diversas perspectivas y 3) contrastar la política pública en educación con las acciones-actividades que llevan a cabo las universidades.

\section{Referencias}

Acosta, A. (2015). La fimpes y la mejora de la calidad de instituciones privadas: Un estudio acerca del concepto de calidad y de los procesos de acreditación en tres universidades particulares. Revista de la educación superior, 44(175), 169-175. Recuperado de https://www.redalyc.org/articulo.oa?id=60445662008.

Aguila, V. (2005). El concepto calidad en la educación universitaria: clave para el logro de la competitividad institucional. Revista Iberoamericana de Educación, 36(12), 1-7. Recuperado de https://doi.org/10.35362/rie36122886.

Alvarado, E., Luyando, J. y Picazzo, E. (2015). Percepción de los estudiantes sobre la calidad de las universidades privadas en Monterrey. Revista Iberoamericana de Educación Superior, 6(17), 58-76. Recuperado de https://doi.org/10.1016/j.rides.2015.10.003.

Alvarado, E., Morales, D. y Aguayo, E. (2016). Percepción de la calidad educativa: caso aplicado a estudiantes de la Universidad Autónoma de Nuevo León y del Instituto Tecnológico de Estudios Superiores de Monterrey. Revista de la Educación Superior, $\quad 45(180), \quad$ 55-74. Recuperado de https://doi.org/10.1016/j.resu.2016.06.006.

Andreasen, M., Colombo, M., Mollo, G., Gilli, J. y López, M. (2015). Calidad de la educación superior: percepciones de los estudiantes universitarios argentinos. Gestão Universitária na América Latina, 8(4), 101-116. Recuperado de https://www.redalyc.org/articulo.oa?id=319343257007.

Araya, L. (2013). ¿Qué hemos aprendido sobre la calidad de servicio en educación superior?. Revista Pilquen, 2(16), 1-12. Recuperado de https://www.redalyc.org/articulo.oa?id=347532061008. 


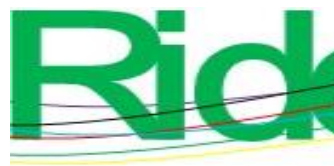

Revista Iberoamericana para la Investigación y el Desarrollo Educativo ISSN $2007-7467$

Avendaño, W., Paz, L. y Parada, A. (2016). Estudio de los factores de calidad educativa en diferentes instituciones educativas de Cúcuta. Investigación \& Desarrollo, 24(2), 329-354.

Recuperado de https://www.redalyc.org/articulo.oa?id=26850086006.

Bernasconi, A. y Rodríguez, E. (2018). Análisis exploratorio de las percepciones sobre los estilos de liderazgo, el clima académico y la calidad de la formación de pregrado. Formación Universitaria, 11(3), 29-40. Recuperado de https://dx.doi.org/10.4067/S0718-50062018000300029.

Bianchetti, R. (2009). Educación de calidad: uno de los dilemas fundamentales para las políticas educativas que se propongan demostrar, que "Otro Mundo es Posible". Referencias. Boletín del Foro Latinoamericano de Políticas Educativas, 26(6), 129

Recuperado de https:// http://bibliotecavirtual.clacso.org.ar/Argentina/lpp/20100426081918/2.pdf.

Bianchetti, A. (2017). Calidad educativa: concepciones y debate. Revista Electrónica de Investigación Educativa, 19(1), 1-3. Recuperado de http://redie.uabc.mx/redie/article/view/1502.

Clara, M. y Vega, C. (2020). La noción de educación de calidad a nivel superior: una reflexión crítica desde el discurso del desarrollo. Interconectando Saberes, 10(5), 67-82. Recuperado de https://doi.org/10.25009/is.v0i10.2672.

Cubillo, M. y Rozo, D. (2009). El concepto de calidad: Historia, evolución e importancia para la competitividad. Revista de la Universidad de la Salle (48), 80-99. Recuperado de https://ciencia.lasalle.edu.co/ruls/vol2009/iss48/4/.

Duque, E. y Chaparro, C. (2012). Medición de la percepción de la calidad del servicio de educación por parte de los estudiantes de la UPTC Duitama. Criterio Libre, 10(16), 159-192. Recuperado de https://doi.org/10.18041/19000642/criteriolibre.2012v10n16.1168.

Fernández, F., Egido, I. and Carballo, R. (2016). Impact of quality management systems on teaching-learning processes. Quality Assurance in Education, 24(3), 394-415. Retrieved from https://10.1108/QAE-09-2013-0037.

Garzón, A. (2013). La mejora continua y la calidad en instituciones de formación profesional. El proceso de enseñanza-aprendizaje. (Tesis de doctorado). Universidad Autónoma de Barcelona, Bellaterra. Recuperado de http://hdl.handle.net/10803/96828.

Garzón, A. (2018) Modelo para el seguimiento y acompañamiento a graduados (SAG), una visión holística de la gestión de la calidad de la educación superior: a holistic 


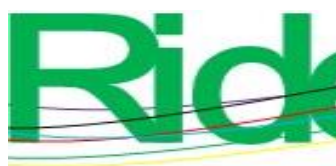

Revista Iberoamericana para la Investigación y el Desarrollo Educativo ISSN $2007-7467$

view of quality management in higher education. Educación, 27(52), 201-218. Recuperado de https://dx.doi.org/10.18800/educacion.201801.011.

González, L. y Espinoza, Ó. (2008). Calidad en la educación superior: concepto y modelos. Calidad en la Educación, (28), 248-276. Recuperado de https://doi.org/10.31619/caledu.n28.210.

Greenacre, M. (2008). La práctica del análisis de correspondencias. Bilbao, España: Fundación BBVA.

Harvey, L. and Green, D. (1993). Defining Quality. Assessment \& Evaluation in Higher Education, $\quad$ 18(1), $\quad 9-34 . \quad$ Retrieved from https://doi.org/10.1080/0260293930180102.

Harvey, L. and Stensaker, B. (2008). Quality Culture: understandings, boundaries and linkages. European Journal of Education, 43(4), 427-442. Retrieved from https://doi.org/10.1111/j.1465-3435.2008.00367.x.

Hernández, R., Fernández, C. y Baptista, M. (2014). Metodología de la investigación. México: McGraw-Hill.

Lago, D., Gamoba, A. y Montes, A. (2014). Calidad de la educación superior: un análisis de sus principales determinantes. Saber, Ciencia y Libertad, 9(1), 157-170. Recuperado de https://doi.org/10.18041/2382-3240/saber.2014v9n1.2006.

López, N. (2007) Las nuevas leyes de educación en América Latina. Una lectura a la luz. del panorama social y educativo de la región. Buenos Aires, Argentina: Instituto Internacional de Planeamiento de la Educación de la Unesco, Sede Buenos Aires.

Lorente, M. (2016). Claves de la calidad de la educación en América Latina. En Valle, J. y Nuñes, J. (coords.), Educación, supranacionalidad y ciudadanía (pp. 11-27). Madrid, España: Fundación Santillana.

Lugo, M. T. y Ithurburu, V. (2019). Políticas digitales en América Latina. Tecnologías para fortalecer la educación de calidad. Revista Iberoamericana de Educación, 79(1), 11-31. Recuperado de https://doi.org/10.35362/rie7913398.

Mayo, J. C., Loredo, C. N. y Reyes, S. N. (2015). En torno al concepto de calidad. Reflexiones para su definición. Retos de la Dirección, 9(2), 49-67. Recuperado de http://scielo.sld.cu/scielo.php?script=sci_arttext\&pid=S230691552015000200004\&lng=es\&tlng=pt.

Mendoza, F. y Ortegon, M. (2019). La evaluación en educación superior con fines de acreditación de alta calidad a través de un modelo sistémico con teoría de redes. Revista de la Educación Superior, 48(192), 1-21. Recuperado de http://resu.anuies.mx/ojs/index.php/resu/article/view/925/377. 
Murillo, F. y Román, M. (2010). Retos en la evaluación de la calidad de la educación en América Latina. Revista Iberoamericana de Educación, (53), 97-120. Recuperado de http://hdl.handle.net/11162/24200.

Orozco, J., Olaya, A. y Villate, V. (2009). ¿Calidad de la educación o educación de calidad? Una preocupación más allá del mercado. Revista Iberoamericana de Educación, (51), 161-181. Recuperado de https://doi.org/10.35362/rie510637.

Otzen, S. y Manterola, C. (2017). Técnicas de muestreo sobre una población a estudio. International Journal of Morphology, 1(35), 227-232. Recuperado de http://dx.doi.org/10.4067/S0717-95022017000100037.

Pérez, R., López, F., Peralta, M. y Municio, P. (2000). Hacia una educación de calidad. Gestión,instrumentos y evaluación. Madrid, España: Narcea.

Schiefelbein, E. y Schiefelbein, P. (2000). Determinantes de la calidad: ¿qué falta mejorar?. Perspectivas, 4(1), 37-64. Recuperado de http://www.dii.uchile.cl/ Revista/ArticulosVol4-N1/Schiefelbein-EyP.pdf.

Supo, J. (2012). Cómo validar un instrumento. La guía para validar un instrumento en 10 pasos. Lima, Perú: Biblioteca Nacional del Perú.

Salas, I. (2013). La acreditación de la calidad educativa y la percepción de su impacto en la gestión académica: el caso de una institución del sector no universitario en México. Calidad en la Educación, (38), 305-333. Recuperado de https://dx.doi.org/10.4067/S0718-45652013000100009. 


\begin{tabular}{|c|c|}
\hline Rol de Contribución & Autor (es) \\
\hline Conceptualización & $\begin{array}{l}\text { Miguel Ángel Clara Zafra (principal), César Vega Zárate } \\
\text { (Igual) }\end{array}$ \\
\hline Metodología & Miguel Ángel Clara Zafra (principal) \\
\hline Software & Miguel Ángel Clara Zafra (principal) \\
\hline Validación & César Vega Zárate (principal) \\
\hline Análisis Formal & Miguel Ángel Clara Zafra (principal) \\
\hline Investigación & $\begin{array}{l}\text { Miguel Ángel Clara Zafra (principal), César Vega Zárate } \\
\text { (Igual) }\end{array}$ \\
\hline Recursos & César Vega Zárate (Igual) \\
\hline Curación de datos & $\begin{array}{l}\text { Miguel Ángel Clara Zafra (principal), César Vega Zárate } \\
\text { (Igual) }\end{array}$ \\
\hline $\begin{array}{l}\text { Escritura - Preparación del } \\
\text { borrador original }\end{array}$ & Miguel Ángel Clara Zafra (principal) \\
\hline $\begin{array}{l}\text { Escritura - Revisión y } \\
\text { edición }\end{array}$ & $\begin{array}{l}\text { César Vega Zárate (principal), Miguel Ángel Clara Zafra } \\
\text { (Igual) }\end{array}$ \\
\hline Visualización & $\begin{array}{l}\text { Miguel Ángel Clara Zafra (principal), César Vega Zárate } \\
\text { (Igual) }\end{array}$ \\
\hline Supervisión & Miguel Ángel Clara Zafra, César Vega Zárate (Igual) \\
\hline Administración de Proyectos & César Vega Zárate (principal) \\
\hline Adquisición de fondos & $\begin{array}{l}\text { César Vega Zárate (principal), Miguel Ángel Clara Zafra } \\
\text { (Igual) }\end{array}$ \\
\hline
\end{tabular}

